\title{
Survivorship Care Planning: Why Is It Taking So Long?
}

\author{
Sarah A. Birken, PhD, a,b and Deborah K. Mayer, PhD, RN, AOCN ${ }^{b}$
}

\begin{abstract}
Survivorship care planning is a process that focuses on the transition from active cancer treatment to a coordinated and comprehensive care system to address patients' unique needs. Survivorship care plans (SCPs) are increasingly viewed as a key component of survivorship care planning. SCPs have been recommended for $>10$ years to promote care coordination, communication, and record-keeping efficiency; however, SCP implementation has been challenging. We elaborate on the challenges of SCP implementation, summarize evidence regarding potential methods of promoting SCP implementation, and describe a research agenda for generating more definitive evidence to support SCP implementation in practice.
\end{abstract}

Attention to survivorship issues has grown since the formation of the National Coalition for Cancer Survivorship in 1986 and the establishment of the NCI's Office of Cancer Survivorship in 1996. In 2006, the Institute of Medicine (IOM) published the seminal report, "From Cancer Patient to Cancer Survivor: Lost in Transition," which identified the transition from active treatment to life after treatment as a time fraught with problems for survivors seeking and obtaining appropriate, high-quality care. ${ }^{1}$ Why does this matter? The number of people living with or beyond cancer has increased from 3.6 million in 1975 to a projected 26 million by 2040. ${ }^{2}$ Cancer survivors have unique healthcare needs. For example, cancer survivors are at risk for late effects of cancer and its treatment, such as organ toxicity and developing new cancers. ${ }^{3}$ Concerns exist about the ability to deliver care to address these needs in an evolving healthcare environment, with a primary care and oncology workforce that is not keeping pace with demand. ${ }^{4}$

Survivorship care planning is a process that focuses on the transition from active cancer treatment to a

From a Department of Health Policy and Management, Gillings School of Global Public Health, and ' $U N C$ Lineberger Comprehensive Cancer Center, The University of North Carolina at Chapel Hill, Chapel Hill, North Carolina. Submitted March 29, 2017; accepted for publication June 15, 2017.

The authors have disclosed that they have no financial interests, arrange- coordinated and comprehensive care system to address patients' unique needs. Survivorship care plans (SCPs) are increasingly viewed as a key component of survivorship care planning. The IOM's "Lost in Transition" report ${ }^{1}$ recommended that "patients completing primary treatment should be provided with a comprehensive care summary and follow-up plan that is clearly and effectively explained." The objective of the IOM's recommendation was to promote care coordination, communication, and record-keeping efficiency. Although there are other survivors with active disease, this review focuses on patients ending curative treatment.

Following the IOM's recommendation, several high-profile organizations have advocated for SCP implementation. Perhaps most notably, based on the IOM's recommendation, in 2012 the American College of Surgeons Commission on Cancer (CoC), the largest cancer program accrediting body in the United States, developed Standard 3.3, which required accredited cancer programs to establish a process for developing and delivering SCPs to all patients ending cancer

ments, affiliations, or commercial interests with the manufacturers of any products discussed in this article or their competitors.

Correspondence: Deborah K. Mayer, PhD, RN, AOCN, UNC Lineberger Comprehensive Cancer Center, University of North Carolina, Campus Box 7460, Chapel Hill, NC 27516. E-mail: dmayer@unc.edu 
treatment. ${ }^{5}$ Despite these efforts, SCP implementation in the United States remains at an estimated $12 \%$ to $43 \% .^{6-10}$ Salz et $\mathrm{al}^{9}$ found that $43 \%$ of NCIdesignated cancer programs used SCPs for breast cancer survivors. In their study of Massachusetts cancer care providers, Merport et $\mathrm{al}^{10}$ found that just $14 \%$ developed SCPs. Additionally, $<10 \%$ of the nationally representative sample of medical oncologists in the 2013 study by Forsythe et $\mathrm{a}^{11}$ reported always or almost always developing SCPs for survivors. Therefore, the objective of this review is to elaborate on the challenges of SCP implementation, summarize evidence regarding potential strategies for promoting SCP implementation, and describe a research agenda for generating more definitive evidence to support SCP implementation in practice.

\section{The Challenge of SCP Implementation}

Table 1 summarizes evidence of the challenge of SCP implementation. Evidence suggests that cancer care providers often do not develop SCPs; when SCPs are developed, they frequently contain clinically significant errors or omissions ${ }^{12}$ (see Table 2 for SCP components recommended by ASCO and the $\mathrm{CoC})$, and they are seldom delivered to survivors or their primary care physicians (PCPs) ${ }^{6-9}$ Birken et $\mathrm{al}^{7}$ found that more than half of surveyed cancer programs developed SCPs for at least $50 \%$ of survivors; however, the SCPs were delivered to just $15 \%$ of the survivors and just 23\% of their PCPs. A 2016 study of SCPs developed in 2 Midwestern cancer programs found that $45 \%$ contained $\geq 1$ omissions (eg, dates, auxiliary surgeries, receptors) and $46 \%$ contained errors (eg, discrepancies in dates, stage, surgeries, chemotherapy, receptors) ${ }^{12}$

The challenge of implementing SCPs identified in research has been underscored in practice. In response to concerns raised in a survey of accredited programs regarding readiness to implement $\mathrm{SCPs}^{13}$ and work from ASCO addressing the challenges of implementation, ${ }^{14}$ the $\mathrm{CoC}$ revised its 2012 standard to phase in SCP implementation, covering $10 \%$ of eligible patients in 2015, 25\% in 2016, 50\% in 2017, $75 \%$ in 2018 , and $100 \%$ in $2019 .{ }^{15}$

\section{Evidence of Determinants of SCP Implementation}

A SCOPUS review conducted on studies published between 2006 to 2016 identified 297 publications with a focus on SCPs (Figure 1). Although this reflects increased attention, little advancement has been made in implementing SCPs. A growing body of evidence catalogues barriers to SCP implementation. In the earliest studies, identified barriers were tangential to primary outcomes. For example, in the 2012 study by Merport et al, ${ }^{10}$ Massachusetts cancer care providers reported that barriers to SCP implementation included lack of training, reimbursement, and templates. Many other early studies of barriers to SCP implementation relied primarily on anecdotal evidence as a basis for identifying barriers. These studies focused primarily on the availability of time,

\section{Table 1. Challenges to Implementing SCPs}

\begin{tabular}{|c|c|c|}
\hline Study & Key Outcome & Findings ( $\%$ of Respondents) \\
\hline Birken et al, ${ }^{8} 2013$ & Prevalence and barriers to SCP use & $\begin{array}{l}<25 \% \text { of providers in cancer programs had ever used an SCP }(76 \%) \\
\text { SCPs used for }<50 \% \text { of survivors } \\
\text { SCPs delivered to } \leq 50 \% \text { of survivors }(62 \%) \\
\text { SCPs delivered to } \leq 25 \% \text { of PCPs }(50 \%)\end{array}$ \\
\hline Birken et al, ${ }^{6} 2014$ & SCP use & $\begin{array}{l}\text { SCPs used }(44 \%) \\
\text { SCPs delivered to } \leq 50 \% \text { survivors }(63 \%) \\
\text { SCPs delivered to } \leq 50 \% \text { PCPs }(61 \%)\end{array}$ \\
\hline Birken et al,? 2014 & SCP development and delivery & $\begin{array}{l}\text { SCPs developed for } \geq 75 \% \text { of survivors ( } 15 \% \text { of programs) } \\
\text { SCPs delivered to } \geq 75 \% \text { of survivors }(25 \%) \\
\text { SCPs delivered to } \geq 75 \% \text { of PCPs }(23 \%) \\
\text { All } 3 \text { criteria above met }(4 \%)\end{array}$ \\
\hline Salz et al, ${ }^{9} 2012$ & $\begin{array}{l}\text { Institutional adoption and survivor } \\
\text { receipt of SCPs }\end{array}$ & $\begin{array}{l}\text { In } 52 \% \text { of } \mathrm{NCl} \text {-designated Comprehensive Cancer Centers, }<50 \% \text { of survivors } \\
\text { received SCPs }\end{array}$ \\
\hline Tevaarwerk et al, ${ }^{12} 2017$ & $\begin{array}{l}\text { Prevalence and types of error/ } \\
\text { omission in treatment summaries }\end{array}$ & $\begin{array}{l}\geq 1 \text { omissions: } 30 \% \text { of EHR-based and } 15 \% \text { of software-based summaries } \\
\geq 1 \text { errors: } 10 \% \text { of EHR-based and } 46 \% \text { of software-based summaries } \\
\text { Types of omissions: dates, axillary surgeries, specification of receptors } \\
\text { Types of errors: dates, stage, surgeries, chemotherapy, receptors }\end{array}$ \\
\hline
\end{tabular}

Abbreviations: EHR, electronic health record; PCPs, primary care physicians; SCPs, survivorship care plans. 


\section{Table 2. SCP Components Recommended by ASCO} and the Commission on Cancer

- Contact information for oncology team members and location of treatment facility

- Name of ongoing adjuvant therapy, expected duration, and anticipated adverse effects

- Schedule of follow-up-related clinical visits (including frequency, location, and provider)

- Schedule of cancer surveillance testing for recurrence (including frequency, location, and provider)

- Schedule for screening of new primary cancers (if different from general population recommendations; including frequency, location, and provider)

- Schedule of noncancer periodic testing and screening visits (including frequency, location, and provider)

- List of possible symptoms of cancer recurrence/general statement encouraging patient to seek medical care for any new or unusual symptoms

- List of rare but clinically significant late and/or long-term effects based on individual survivors' diagnosis and treatment history

- List of resources (national and local) pertaining to other items with which survivors have experienced difficulties (eg, mental health, employment, insurance)

- General statement emphasizing importance of maintaining healthy diet, exercising regularly, reducing alcohol consumption, and quitting smoking

Abbreviation: SCP, survivorship care plan.

Data from Mayer DK, Nekhlyudov L, Snyder CF, et al. American Society of Clinical Oncology clinical expert statement on cancer survivorship care planning. J Oncol Pract 2014;10:345-351.

reimbursement, and information technology for SCP use. ${ }^{6-8,10,16}$

Other studies have suggested that lack of guidance may limit SCP implementation. One found that extant guidelines for SCP use are generally of poor quality, and suggested this may represent a barrier to implementation. ${ }^{17}$ Another study emphasized that consensus is lacking regarding which cancer

A

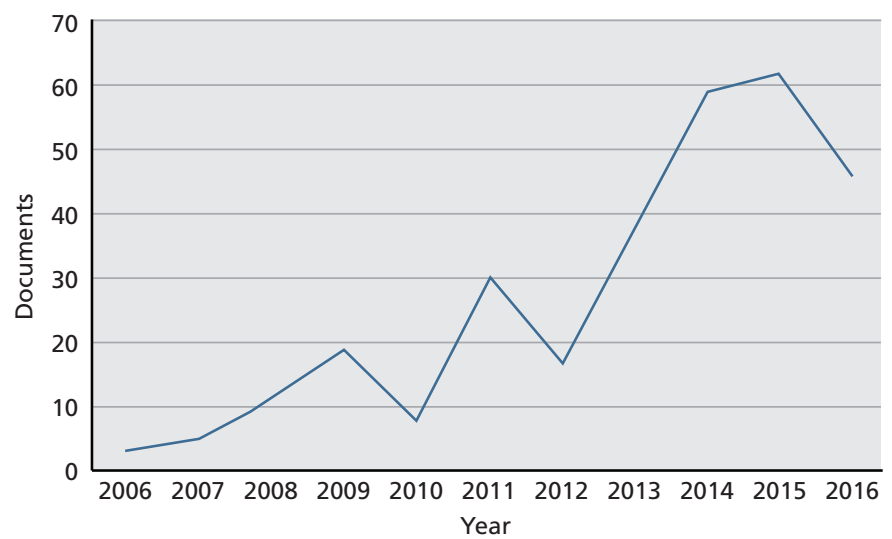

care providers are best-suited to develop SCPs, ${ }^{18}$ and that cancer care providers want to use SCPs but lack the resources and skills necessary to do so. ${ }^{19}$

Perhaps as a result of the aforementioned barriers, cancer programs often implement SCPs with the goal of maintaining accreditation rather than improving survivorship care. ${ }^{6}$ Including SCPs in patient health records may count toward meeting accreditation standards, but SCPs must also be delivered with recommended content to benefit survivors and PCPs and to address the intent of CoC Standard 3.3.

Aside from scholars' recommendations to avoid the barriers identified in their studies, limited evidence exists regarding facilitators of SCP implementation. One interview study found that cancer care providers were inclined to use SCPs when they believed that doing so would have positive outcomes (eg, benefitting survivors, providers, and the healthcare system as a whole), did not compete with other demands for their time, was feasible in their organization (eg, they could deliver SCPs at a dedicated visit, had information technology that facilitated SCP use), and was a priority among their peers and their department or organization. ${ }^{20}$ Practical recommendations for facilitating SCP use are consistent with these findings. Participants in the IOM's 2007 Implementing Cancer Survivorship Care Planning Workshop ${ }^{21}$ warned that a "profound cultural shift will be required to change entrenched practice patterns" to facilitate SCP implementation. Earle ${ }^{22}$ echoed this sentiment, stating, "a culture change is necessary so that providers recognize survivorship care planning

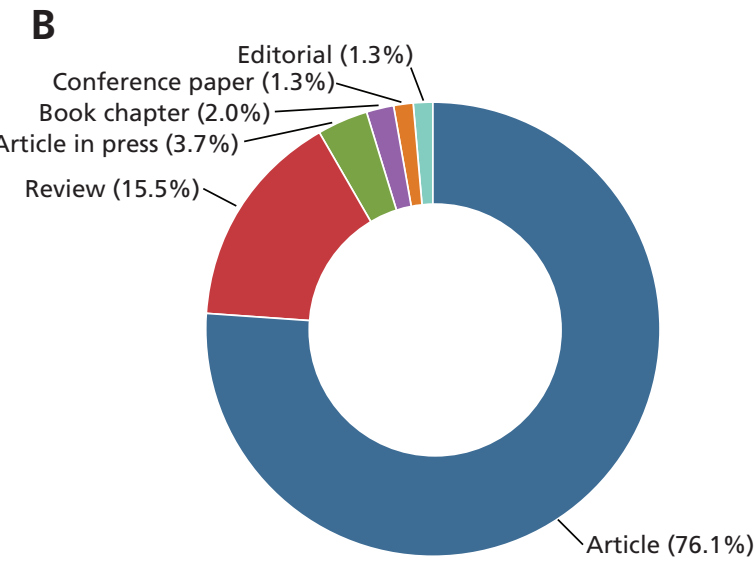

Figure 1. SCOPUS survivorship care plan review by (A) numbers of documents per year and (B) documents by type (2006-2016). ${ }^{\text {a }}$ aArticles with "survivorship care plan" in title or abstract. 
as a standard and expected component of quality cancer care and so that patients recognize the importance of posttreatment care and adherence to followup recommendations." The extent to which this culture shift has occurred is unclear.

Efforts have been made to facilitate implementation in practice, many of which have involved developing and refining SCP templates. ASCO created a general SCP template, as well as a number of diseasespecific SCPs ${ }^{23}$; in 2007, a Prescription for Living template for cancer survivors was published ${ }^{24}$; and in 2008, the University of Pennsylvania developed an online OncoLife Survivorship Care Plan ${ }^{25}$ and Journey Forward created an online SCP builder ("Cancer Care Plan Builder"). ${ }^{26}$ In its 2016 Cancer Program Standards, the CoC provided greater detail than in previous standards in defining patient eligibility criteria and expanding on the process descriptions and expected outcomes. ${ }^{27}$ Whether these practical efforts have facilitated implementation remains unclear.

\section{Future Directions for Facilitating SCP Implementation in Practice}

Applying implementation science to SCP research and practice is a critical next step to identify facilitators of implementation. As demonstrated in some extant research, ${ }^{17}$ implementation science provides frameworks and strategies to help researchers consider the context of SCP use to identify facilitators of implementation. ${ }^{28}$ Implementation science can promote understanding of what works, where, and why with respect to SCPs, contributing to meaningful practice change.

Currently funded, yet-unpublished studies are making strides toward identifying strategies for SCP implementation. In a study conducted by the Institute for Patient-Centered Initiatives and Health Equity at George Washington University Cancer Center, researchers surveyed cancer care providers regarding their processes for SCP implementation, with the long-term goal of identifying SCP implementation models that may be linked to improved communication and coordination among survivors, cancer care providers, and follow-up care providers (ie, best practices). A North Carolina Translational and Clinical Sciences Institute-funded study used qualitative comparative analysis (QCA) to identify strategies that promote SCP implementation among cancer programs participating in the ASCO Quality Oncology Practice Initiative. QCA begins with interviews to identify successful SCP implementation strategies to be assessed in a future comparative effectiveness trial. In a randomized controlled trial funded by the Patient-Centered Outcomes Research Institute (PCORI), researchers will test 3 strategies for SCP implementation, with the goal of identifying strategies that are feasible, patient-centered, and effective in increasing SCP development and delivery. Several of these studies are promising because they take into account stakeholders' perspectives on SCP implementation. This is critical because effective implementation is likely to depend on stakeholder engagement (eg, cancer care providers delivering SCPs in a way that conveys the content to survivors and PCPs).

\section{Summary and Discussion}

For more than 30 years, survivorship has gained recognition as a component of cancer care, yet it still has not become a standard of care; this exceeds previous studies of how long it takes for evidence to be implemented in practice (17 years). ${ }^{29}$ Knowledge to date suggests successful SCP implementation likely requires culture change. That is, instead of viewing SCPs as a requirement to be met, they should be viewed as an opportunity to develop systems for delivering comprehensive survivorship care, of which SCPs are just one component. Opportunities for developing survivorship care systems might include (but are not limited to) establishing referral networks through partnerships with PCPs with a special interest in caring for survivors; identifying high-risk survivors and those with complex needs; reallocating resources to ensure that SCPs are not just developed but also reviewed with survivors; promoting provider education through continuing education; and continuously improving survivorship care systems. Future studies should assess stakeholders' perspectives on survivorship care systems' effectiveness (ie, the extent to which stakeholders perceive survivorship care systems as meeting their needs). Finally, recognizing cancer programs' diversity (eg, patient, staffing, and payer mix), future studies should investigate approaches to adapting and tailoring strategies for developing effective survivorship care systems, including SCPs, to fit cancer programs' unique contexts. 


\section{References}

1. Institute of Medicine. From Cancer Patient to Cancer Survivor: Lost in Transition. Available at: https://www.nap.edu/catalog/11468/from-cancerpatient-to-cancer-survivor-lost-in-transition. Accessed March 28, 2017.

2. Bluethmann SM, Mariotto AB, Rowland JH. Anticipating the "silver tsunami": prevalence trajectories and comorbidity burden among older cancer survivors in the United States. Cancer Epidemiol Biomarkers Prev 2016;25:1029-1036.

3. American Cancer Society. Cancer Treatment \& Survivorship Facts \& Figures 2016-2017. Available at: https://www.cancer.org/content/dam/ cancer-org/research/cancer-facts-and-statistics/cancer-treatment-andsurvivorship-facts-and-figures/cancer-treatment-and-survivorship-factsand-figures-2016-2017.pdf. Accessed March 28, 2017.

4. Jacobs LA, Shulman LN. Follow-up care of cancer survivor: challenges and solutions. Lancet Oncol 2017;18:e19-29.

5. American College of Surgeons Commission on Cancer. Cancer Program Standards 2012: Ensuring Patient-Centered Care. Version 1.2.1. Available at: https://www.facs.org/ /media/files/quality\%20programs/cancer/coc/ programstandards2012.ashx. Accessed March 27, 2017.

6. Birken SA, Deal AM, Mayer DK, Weiner BJ. Determinants of survivorship care plan use in United States programs. J Canc Educ 2014;29:720-727.

7. Birken SA, Deal AM, Mayer DK, Weiner BJ. Following through: the consistency of survivorship care plan use in United States cancer programs. J Cancer Educ 2014;29:689-697.

8. Birken SA, Mayer DK, Weiner BJ. Survivorship care plans: prevalence and barriers to use. J Canc Educ 2013;28:290-296.

9. Salz T, Oeffinger KC, McCabe MS, et al. Survivorship care plans in research and practice. CA Cancer J Clin 2012;62:101-117.

10. Merport A, Lemon SC, Nyambose J, Prout MN. The use of cancer treatment summaries and care plans among Massachusetts physicians. Support Care Cancer 2012;20:1579-1584.

11. Forsythe LP, Parry C, Alfano CM, et al. Gaps in survivorship care plan delivery and potential benefits to survivorship care [abstract]. J Clin Oncol 2013:31(Suppl):Abstract 9594.

12. Tevaarwerk AJ, Hocking WG, Zeal JL, et al. Accuracy and thoroughness of treatment summaries provided as part of survivorship care plans prepared by two cancer centers. J Oncol Pract 2017;13:e486-495.

13. American College of Surgeons. Accreditation Committee Clarifications for Standard 3.3 Survivorship Care Plan. Available at: https://www. facs.org/publications/newsletters/coc-source/special-source/standard33. Accessed March 27, 2017.

14. Mayer DK, Nekhlyudov L, Snyder CF, et al. American Society of Clinical Oncology clinical expert statement on cancer survivorship care planning. J Oncol Pract 2014;10:345-351.
15. Shulman LN, Ferris L, Takanishi DM, McKellar D. Treatment summaries and survivorship care plans: the approach by the Commission on Cancer to increase use. J Oncol Pract 2015;11:40-41.

16. Hewitt M, Bamundo A, Day R. Perspectives on post-treatment cancer care: qualitative research with survivors, nurses, and physicians. J Clin Oncol 2007;25:2270-2273.

17. Birken SA, Ellis SD, Walker JS, et al. Guidelines for the use of survivorship care plans: a systematic quality appraisal using the AGREE II instrument. Implement Sci 2015;10:63.

18. Keesing S, McNamara B, Rosenwax L. Cancer survivors' experiences of using survivorship care plans: a systematic review of qualitative studies. J Cancer Surviv 2015;9:260-268.

19. Klemanski DL, Browning KK, Kue J. Survivorship care plan preferences of cancer survivors and health care providers: a systematic review and quality appraisal of the evidence. J Cancer Surviv 2016;10:71-86.

20. Birken SA, Presseau J, Ellis SD, et al. Potential determinants of health-care professionals' use of survivorship care plans: a qualitative study using the theoretical domains framework. Implement Sci 2014;9:167.

21. A National Coalition for Cancer Survivorship and Institute of Medicine National Cancer Policy Forum Workshop. Implementing Cancer Survivorship Care Planning: Workshop Summary. Washington, DC: National Academies Press; 2007.

22. Earle CC. Failing to plan is planning to fail: improving the quality of care with survivorship care plans. J Clin Oncol 2006;24:5112-5116.

23. American Society of Clinical Oncology. Survivorship Care Planning Tools. Available at: http://www.asco.org/practice-guidelines/cancer-careinitiatives/prevention-survivorship/survivorship-compendium. Accessed March 28, 2017.

24. Haylock PJ, Mitchell SA, Cox T, et al. The cancer survivor's prescription for living. Am J Nurs 2007;107:58-70.

25. OncoLink. OncoLife Survivorship Care Plan. Available at: https://www. oncolink.org/oncolife/. Accessed March 28, 2017.

26. Journey Forward. Treatment Plan. Available at: https://www. journeyforward.org. Accessed March 28, 2017.

27. American College of Surgeons Commission on Cancer. Cancer Program Standards: Ensuring Patient-Centered Care. Available at: https://www. facs.org/ /media/files/quality $\% 20$ programs/cancer/coc/2016\%20coc $\% 20$ standards\%20manual_interactive\%20pdf.ashx. Accessed March 28, 2017.

28. Parry C, Kent EE, Forsythe LP, et al. Can't see the forest for the care plan: a call to revisit the context of care planning. J Clin Oncol 2013;31:26512653.

29. Morris ZS, Wooding S, Grant J. The answer is 17 years, what is the question: understanding time lags in translational research. J R Soc Med 2011;104:510-520. 